\section{CASE OF EXTRA-UTERINE GESTATION.}

\section{By C. E. GOOdING, M.D. McGitil Coli., M.R.C.S.}

UNFORTUNATELY cases of extra-uterine gestation do not occur so rarely as perhaps to warrant a single instance being put on record by itself. It is only on account of some specially interesting features which it presented that $I$ venture to report the following case.

Early in December, 1885, I was called to a Quadroon woman, aged forty-nine and unmarried. She was suffering from well-marked symptoms of acute cystitis, following apparently on exposure to cold and damp a few days before. She also complained of a constant and severe pain, with great tenderness in the right iliac region, shooting thence down the thigh of the same side. Some fulness and diminished resonance on percussion could be detected in the right iliac fossa, and extending slightly beyond the middle line of the abdomen. Conjoined vaginal examination revealed a hard, non-fluctuating, and very sensitive swelling, about the sizg of a coconut, rising out of the pelvis on the right side. The uterus, which was of normal depth, was pushed over to the left, but moved separately from the tumour. The latter was diagnosed as ovarian in its origin, and from its density probably fibroid or dermoid. Under appropriate treatment the vesical symptoms entirely subsided in a couple of weeks, and the patient was then lost sight of for some time, although told to report herself, should other symptoms due to the presence of the tumour develop. Thirteen months after, in January, 1887, she was admitted under my care in the St. Philip's Almshouse. She brought with her three bodies slightly encrusted with phosphates, which she had passed per urethram during the previous week, and which were at once recognised as the tibia and two ribs of a child apparently at or near full term. I learnt then that since I had seen her she had had several attacks of cystitis, and been under the care of more than one medical man, with but very partial relief to her symptoms. The following history was now elicited : Began to menstruate at fourteen, and was regular until twenty, when her first and only child was born. With the exception of very slight occasional intermissions, she continued regular until ten years ago. Her menses then ceased for five or six months, but although she had exposed herself to the chances of conception she did not consider herself pregnent. She declares positively that she did not observe any of the usual signs of pregnancy, but admits that she was unusually fat about this period. Hrom this time, however, she has never felt strong, being easily tired and very subject to pains in the back and lower part of abdomen, and running down the leg on the right side. After this interruption her menses returned and were fairly regular until they ceased entirely three years ago.

The woman's condition on admission was as follows:She has become very much emaciated since last seen; is suffering with chronic cystitis; micturition very frequent, and the act accompanied with intense pain; bladder when sounded is found to contain a large number of bones; dulness over tumour has lessened in extent, and tumour itself per vaginam feels more soft and yielding; is suffering with hectic, and has an evening temperature in the neighbourhood of $101^{\circ}$

She was ordered tonics and good diet, and the bladder to be washed out frequently with antiseptic injections. Under this treatment her general condition improved, and after two or three wesks (during which time she had passed a couple more small bones), on consultation with Dr. Archer I decided to operate. This was done on Februsry 12tb, Dr. Archer assisting; Dr. Greaves administered ether, and Dr. Shepherd also was present. No injection was used to distend the bladder on account of the manifest communication existing between that viscus and the fotal cyst. The urethra was first dilated until it admitted the index finger, and the bladder was then opened by an incision in the median line above the pubes. With the two index fingers-one passed through the urethra, and the other through the abdominal wound-all the bones were removed through the latter. The bones were found partly in the bladder and partly in a cyst--about the size of the foetal head at term, - which communicated freely with the fundus of the bladder on the right side through a fistulous channel large enough to easily admit the finger. The bladder and cyst were next washed out thoroughly with a full stream of warm carbolised water in order to remove any remaining detritus. The opening into the bladder was left unsuturea, and that night all the urine passed through it.

The next morning a catheter was tied into the bladder through the urethra and retained for one day. After this all the urine passed per urethram, though it was several weeks before complete control over the sphincter vesic was established. After the operation there were no bad symptoms. The bladder was washed out with antiseptic injections untrl its coats had regained their normal condition. In six weoks the abdominal opening had healed all but a little fistula at the upper end, through which a probs could be passed directly backwards for about four inches. A small bit of loose bone was now detected near the bottom, and its removal was soon followed by the healing of the sinus. At the end of three months she was discharged, "feeling better and stronger," as she expressed it, " than she had for many years."

The majority of all cases of extra-uterine gestation end in the death of the mother through rupture of the cyst and consequent hæmorrhage, which usually takes place about the third or fourth month. Sometimes the foetus dies in the early months of pregnancy without rupture of the cyst; then the fluid parts are absorbed and the remainder dries up and becomes quiescent. In a smaller proportion of cases the foetus does not perish until the full term of gestation, and then the ultimate result varies greatly. The fiuid contents of the cyst may dry up, and the foetus (bocoming converted into a substance resembling adipocere) remain quiescent for an indefinite time. On the other hand, the cyst with its contents may undergo suppuration, and the mother die from the violence of the inflammatory process. Should, however, the course of inflammation be less intense, an effort is made to throw off the fotus (which has now become a foreign body) by the formation of a fistulous canal leading from the cyst. This most frequently opens into some part of the intestines, of through the walls of the abdomen, or more rarely into the vagina or bladder. The case recorded above is remarkable, as affording an example of that exceedingly rare termination of an extra-uterine pregnancy-viz., an attempt on the part of nature to effect a cure by getting rid of the contents of the cyst through a fistula into the bladder. It is also interesting from the slight amount of constitutional diso turbance with which the growth of the fotus in its unusual position was accompanied, and from the entire want of those subjective and objective signs of pregnancy, the absence of which prevented the woman and those about her from entertaining the slightest suspi ion of her being pregnant.

\section{THE TREATMENT OF CYSTIC GOITRE. ${ }^{1}$}

BY T. MARK HOVELL, F.R.C.S. EDIN.,

SENIOR SURGEON AND AURAL SURGEON TO THE HOSPITAT FOR DISEASES OF THE THROAT; JUNIOR AURAL SURGEON TO THE LONDOX

HOSPITAL; LECTURER ON DIESASES OF THE THROAT

AT THE LONDON HOSPITAL MEDICAL COLLEGE.

Mr reason for bringing forward a method of treatment by no means new is that, although it is mentioned in all the principal English and American works on Surgery, the prominence it deserves is not accorded to it, and recently cases have been published in which the treatment adoptêd has entailed serious risk to the patient, whose safety would not have been thus unnecessarily jeopardised had the measures I am about to describe been adopted.

The treatment which I advocated is that introduced by Sir Morell Mackenzie in a paper read before the Hunterian Society on Jan. 22nd, 1872, and published by him in THE LANCET of May 11th, 1872 (vol. i., p. 642). ${ }^{2}$ His method briefly consists in tapping the cyst, and when the fluid has been withdrawn injecting a solution of perchloride of iron through the cannula of a syringe and allowing it to remain for a certain time, so as to convert the cyst into a chronic abscess It is essential that the trocar should be introduced at the most dependent part of the tumour to ensure subsequently efficient drainage, upon which greatly depends the success of the operation, and it is an advantage to make the puncture where the cyst wall is thin, provided this condition is found in the position mentioned. Care must be taken to avoid a place traversed by a large vein, and when practicable it is well to introduce the trocar as near the mesial line as possible. The spot having been selected, the skin should

1 Read before the Hunterian Society, April 13th, 1887.
2 See also Birmingham Medical Review, Fol. iv., 1875. 